\title{
Summary Report of the Workshop on the U.S. Use of Surface Waves for Monitoring the CTBT
}

\author{
W.R. Walter \\ M. Ritzwoller
}

September 1, 1998






\section{DISCLAIMER}

This document was prepared as an account of work sponsored by an agency of the Uniled States Government. Neither the United States Government nor the University of California nor any of their employees, makes any warranty, express or implied, or assumes any legal hiability or responsibility for the accuracy, completeness, or usefulness of any information, apparatus, product, or process disclosed, or represents that its use would not infringe privately owned rights. Reference herein to any specific commercial product, process, or service by trade name, trademark, manufacturer, or otherwise, does not necessarily constitute or imply its endorsement, recommendation, or favoring by the United States Government or the University of California. The views and opinions of authors expressed herein do not necessarily state or reflect those of the United States Government or the University of California, and shall not be used for advertising or product endorsement purposes.

This report has been reproduced directly from the best available copy.

Available to DOE and DOE contractors from the

Office of Scientific and Technical Information P.O. Box 62, Oak Ridge, TN 37831

Prices available from (423) 576-8401

Available to the public from the

National Technical Information Service

U.S. Department of Commerce

5285 Port Royal Rd.,

Springfield, VA 22161 


\title{
Summary Report of the \\ Workshop on the U.S. Use of Surface Waves for Monitoring the CTBT.
}

\author{
by W. R. Walter and M. Ritzwoller \\ Lawrence Livermore National Laboratory and U. of Colorado, Boulder
}

\section{Contents:}

I. Overview and Goals of Workshop

page 1 .

II. Summary of Workshop

III. Results and Recommendations

Appendix: Workshop Agenda

\section{Overview and Goals of Workshop}

On March 19 and 20, 1998 a workshop was organized by Mike Ritzwoller and Bill Walter entitled "Workshop on the U.S. Use of Surface Waves in Monitoring a CTBT". It was held at the Department of Physics on the campus of the University of Colorado at Boulder. The workshop addressed the following general research goals of relevance to monitoring and verifying the Comprehensive Test Ban Treaty (CTBT):

A) To apprise participants of current and planned research in order to facilitate information exchange, collaboration, and peer review.

B) To compare and discuss techniques for data selection, measurement, error assessment, modeling methodologies, etc. To compare results in regions where they overlap and understand the causes of observed differences.

C) To hear about the U.S. research customer's (AFTAC and DOE Knowledge Base) current and anticipated interests in surface wave research.

D) To discuss information flow and integration. How can research results be prepared for efficient use and integration into operational systems?

E) To identify and discuss fruitful future directions for research.

This workshop grew out of informal discussions that took place at the 19th annual CTBT Seismic Symposium held in Orlando, Florida in September, 1997 and at the USGS/DOE Location Workshop that occurred in Golden, Colorado in November, 1997. Although the annual CTBT Seismic Symposium adequately addresses research goal A, single topic workshops in which smaller numbers of participants discuss a sharply focused subject in a more intimate, perhaps hands-on, setting, would better address the goals B - E. The USGS/DOE Location Workshop demonstrated the feasibility of this idea.

A full agenda of the workshop is given at the end in an Appendix. 
Surface waves are well suited to be the first topic for such a small CTBT workshop for three reasons.

1) It became apparent at the Orlando meeting that several research groups in academia, industry, the National Laboratories, and AFTAC are working on related research problems in utilizing surface waves in monitoring the CTBT. Although this work is being performed at different scales (regional, continental and global) and in several different (but overlapping) geophysical areas, there is considerable common ground for comparison, peer-review, and, ultimately, consensus on how surface wave measurements are carried out and used for CTBT purposes. To facilitate this, the two Thursday morning sessions were devoted to revealing the results of a series of tests in which the participants engaged prior to the meeting. Participants were asked to supply group velocity measurements for 10 specified event-station pairs as well as a $20 \mathrm{~s}$ Rayleigh wave group velocity map in the region from $10 \mathrm{~N}$ to $50 \mathrm{~N}$ and $25 \mathrm{E}$ to $100 \mathrm{E}$. The purpose was to understand the causes of differences revealed by the comparisons, and to provide a common basis for discussion.

2) It is clear that these disparate research efforts and results need to be integrated with the goal of optimizing the U.S. CTBT monitoring capability. The first Thursday afternoon session was devoted to hearing from the major customers and funders of this research, AFTAC (hosts the NDC), DOE (developing the Knowledge Base), and NTPO/DSWA (funding/managing PRDA contractors).

3) Finally, surface waves have a long-standing role in seismological aspects of treaty monitoring. They play a major role in earthquake-explosion discrimination, via the use of the surface-wave body-wave magnitude ratio (Ms:mb). They have an even longer standing role in providing information about velocity structures in the crust and upper mantle that may be used to improve U.S. capability to locate and characterize seismic events. The remaining sessions in the workshop were designed to address some of these issues.

\section{Summary of Workshop}

The consensus of the participants was that workshop was successful in that it took a positive first step toward achieving its major goals. As discussed later in this report, the fulfillment of the goals of the workshop will require continued cooperative research, data exchange and follow-up meetings in the future.

The first Thursday morning session focused on a comparison of group velocity measurements for a test set of data (see Fig. 1) obtained by different groups. The participating groups were from the University of Colorado (CU, Levshin, Ritzwoller), Los Alamos National Lab (LANL, Jones, Patton), Lawrence Livermore National Lab and associates (LLNL, Walter, Rodgers, Hazler and McNamara), Maxwell (Stevens), and Saint Louis University (SLU, Ammon, Herrmann). The test data set displayed three unanticipated timing errors. Two were in the range from 40 to 50 seconds, which were large enough to induce error but not necessarily be obvious if only the surface waves were examined. Another timing error appeared in a SEED volume received from the Incorporated Research Institutions for Seismology (IRIS) Data Management Center 
(DMC) in February that was absent in a SEED volume received from the same source six months earlier. These problems provoked a general discussion of quality control issues that must be put in place both by research groups and the IRIS DMC.

The comparison of the group velocity measurements obtained by these five groups (see Fig. 2) revealed only small differences, with two key exceptions. The standard deviation of the residuals among the measurements was less than $0.05 \mathrm{~km} / \mathrm{s} \quad(<1.5 \%)$ and the largest differences generally were smaller than $0.1 \mathrm{~km} / \mathrm{s}(<3 \%)$ as shown in Figure 3 . All groups used variations on Gaussian filtering over multiple frequency bands but differed in specifics such as filter width, smoothing and the period limits over which accurate measurement could be obtained. To the extent that these observed differences between different groups values are greater than measurement errors appears to be due to the two exceptions.

First exception, the existence of a systematic bias in the measurements that were made without correcting for the shape of the amplitude spectrum. This effect is only significant for frequencies at which both the spectrum and the group velocity curve have appreciable non-zero slopes. This only occurs away from Airy phases and is most significant at periods above about $25 \mathrm{~s}$ where biases of $1-2 \%$ may occur. Consensus of the participants was that this correction should be made by all the groups.

A second exception was that the groups differed in their choices of the frequency bands over which the measurements were valid. All groups recognize the problems presented at the short period end by Love/Rayleigh interference, multipathing, and scattering, (nicely summarized in a talk by Levshin) but disagreed on exactly where the cuts should be made. Comparison of the curves produced by the groups clearly revealed these issues were the cause of much of the differences at the short period end of the spectrum. Similar discrepancies existed at the long period end of the measured spectra, but were simply related to the decay of the spectrum.

Short period measurements are important because at smaller magnitudes the regional Airy phase (an therefore best surface wave signal-to-noise ratio) often occurs at periods less that $20 \mathrm{~s}$. One method to minimize undesirable propagation effects is to make use of shorter paths for these short periods. In general though there does not appear to be a rapid and simple way to decide where to cut at the short period end of the spectrum without either being overly conservative or risking inclusion of undesirable path effects. The best method, therefore, appears to be redundancy of measurement. In particular, pooling measurements obtained by the group members would allow for clustering, averaging, outlier identification and rejection, and error estimation. This would ultimately place the group velocity maps on a firmer footing than any one institution could provide alone.

The second Thursday morning session presented comparisons of $20 \mathrm{~s}$ Rayleigh wave group velocity maps (CU, LLNL, SLU, Maxwell). Differences were observed and attributed due to methodology, resolution, and variations between the data sets. With one exception, the maps agreed in most regions of good data coverage if differences in intrinsic resolution are taken into consideration. The exception was that the backprojection method with only a few thousand ray patbs underpredicted the amplitudes of the velocity anomalies by a factor of two or more compared with methods that involve a less approximate inversion. It was agreed that the topic of inversion methodologies should be revisited in a smaller Working Group meeting (discussed further below) after group velocity curves are exchanged and common data sets are finalized. 
The first session on Thursday focused on CTBT R\&D customers with much discussion from all the participants. A particular point of discussion was the different requirements and focus of the U.S. NDC compared with the International IDC. Although some contractors contribute to both efforts, which of the research results developed for the NDC may be shared with the IDC remains unresolved at the inter-agency level of the U.S. government. Data flow to the NDC through the DOE Knowledge Base was also discussed. It is important for researchers to provide metadata, essentially documientation about research results in specific form, to accompany products to be considered to be used in the DOE Knowledge Base for use by the U.S. NDC.

Later on Thursday afternoon, there were two presentations (PIDC, McLaughlin; Maxwell, Stevens) on the current use of surface waves at the PIDC, where Maxwell has constructed a global crustal model on a five degree grid from which group velocity curves arc predicted. This model represents perturbations around the modified Mooney CRUST5.1 crustal model and utilized group velocity and phase velocity information from a number of institutions (e.g, CU, Harvard, SLU). The map is currently used to automatically detect and associate surface waves, and measure $20 \mathrm{~s}$ Ms amplitudes. Routine automated path corrected seismic moments and phase-matched filters have been tested and implementation is anticipated in the near future. Many of the complications with building, installing, running, and maintaining semi-automated surface wave software were discussed. The final talk of the day (MIT/BC; Harkrider) evaluated several global group velocity maps at 20 s period using a large data set of nuclear test data and found the map produced by Maxwell and used by the PIDC was better than several older global maps.

The first session on Friday morning focused on 'problems' (e.g., anisotropy, misoriented sensors, group time shifts, off-great-circle path propagation, etc.) posed by the real earth in analyzing surface waves. It was shown that although most of thesc problems strongly affect surface waves at periods longer than about $50 \mathrm{~s}$, at the shorter periods, which are the main focus of for CTBT monitoring ( $5-40$ s period), the effects are not so important. The consensus appeared to be that the main concerns should be misoriented sensors and off-great-circle propagation. The Harvard group (Larson) demonstrated misorientations of 5-15 degrees at a number of stations. Path deviations due to velocity anomalies are well recognized as a source of error near $20 \mathrm{~s}$ period, particularly for long paths (e.g., $>2000 \mathrm{~km}$ ) and are a fruitful area for future work.

The next session on Friday morning concentrated on source mechanism estimation using surface waves. It was shown (LANL, Patton) that the use of global average models, such as PREM, to compute excitation functions may produce systematic errors in scalar moment estimation utilizing stations confined to the continent of the earthquake or explosion. This is particularly important in regions with thick crust and mid-crustal earthquakes (depth $>25 \mathrm{~km}$ ). It was also discussed (Colorado, Levshin) that regional model-and station-based estimates of the moment tensor may be more accurate than global compilations and useful for depth and event discrimination.

Finally, models constructed from surface waves and body waves were discussed in the context of producing improved travel time correction surfaces for improving locations. Discussion centered on how to test and evaluate the structural models. Consensus appeared to be that the regional relocation of Ground Truth events utilizing the 3D structural. information is the litmus test for determining the value of a model. Related questions were 
how to incorporate information from models into a Knowledge Base. These issues define an area of very active research and could very fruitfully be the subject of its own workshop in the future.

In the closing session, the participants indicated that the workshop was very useful. An open exchange of surface wave group velocity curves between the groups was encouraged. It was recognized that by pooling these resources better dispersion maps with more peer-review may be obtained. It was agreed that follow up working group meeting should be held to continue the work begun in this workshop. These meetings should take place after much more exchange of data among the research groups. Participants were asked to submit a few paragraphs and key figures summarizing their presentations for posting on the workshop web site. These presentations are intended to accompany this summary of the workshop after the workshop's conclusion.

\section{Results and Recommendations}

Three main categories of recommendations are identified: dispersion measurement, dispersion tomography, and knowledge transfer. Recommendations in these areas follow.

\section{Dispersion Measurement:}

1. Seismic data quality control needs refinement, systematization, and improvement. Procedures need to be put in place to identify and either discard or correct problems due to errors in timing, station location, orientation, incorrect instrument response, as well as other errors in data received from central data centers such as the IRIS DMC and the IDC. Specific recommendations are the following:

A) It would be very useful if the IRIS DMC would modify and improve its methods of providing data quality information to its customers. Although one of the timing errors in the test data set is listed by the DMC in a table available on the IRIS bulletin board, this information is rather hard to find and use. It is, for example, not tabulated in a data base that allows searching by time of event (which is how data are processed), it is not formatted in reports that are readily readable by both computers and humans, and is not necessarily passed on to the data requesters. In addition, there does not appear to be any data quality information present about non-GSN stations at the IRIS DMC. Better mechanisms for sharing data quality information with researchers and ways for utilizing researcher feedback need to be implemented at the IRIS DMC. In addition, the time dependent changes in data requested from the DMC indicate that a better accounting mechanism for data changes performed at the DMC perhaps need to be implemented.

B) Groups that check timing using P picks, such as LLNL, should report problems to other groups and to the IRIS DMC. The same applies to information about station mislocations, orientations, etc.

2. Group velocity measurements obtained at frequencies with sloped amplitude spectra and sloped group velocity curves are biased unless the shape of the spectrum is taken into consideration. It is recommended researchers investigate and remove this bias in all their measurements. This is already being done by at least two groups. 
3. All CTBT surface wave researchers are encouraged to share their group velocity measurements. This is expected to have the following beneficial effects.

A) It will alert researchers to multipaths, Love/Rayleigh interference and other problems associated with the subjective choice of frequency band over which the measurements are obtained.

B) It will maximize the number of clusters of measurements as well as the number of measurements composing each cluster which will help to provide better error statistics on the measurements, and the rejection of outliers.

C) It will produce the largest possible data set which will help to improve the resolution of the tomographic maps and yield optimal CTB'T monitoring performance.

D) It will reduce duplication of effort and make more effective use of limited resources

4. More group velocity measurements are needed at shorter periods $(\mathrm{T}<20 \mathrm{~s})$ where the Airy phase is most prominent at regional distances for events of smaller magnitude. These measurements should be targeted at specific source regions to provide high accuracy, high resolution calibration for areas expected to generate most of the signals for processing in an operational setting. In order to mitigate the effects of multipathing and attenuation at short periods, measurements should be made on paths of $2000 \mathrm{~km}$ length or less. These "point" measurements for specific source regions complement the tomographic models of group velocity variations. Combining tomographic results and point measurements should provide the most effective calibration for monitoring all potential source regions.

\section{Dispersion Tomography:}

Like all inversions, surface wave tomography is characterized by a number of (largely) subjectively chosen variables such as the inversion methodology, model norm, damping, smoothness constraints, block size, and so on. As with the group velocity measurements, inter-group comparisons of the tomographic maps are encouraged and are expected to bring similar beneficial results. In particular it is recommended that:

1. Surface wave group-velocity based tomographic maps independently produced by different groups should be regularly compared with each other in regions of overlap, and this comparison should be the focus of a future workshop. CTBT performance criteria will need to be developed and invoked to evaluate different tomographic results.

2. The surface wave dispersion tomography results should also be compared with independent geophysical studies and databases such as Pn tomography, waveform modeling, sediment thickness maps, Moho thickness maps, etc. Such data may assist in better defining regional boundaries and extrapolating results when data coverage is poor. It can also improve our confidence in the results. Finally independent geophysical results 
provide valuable constraints when inverting dispersion results for velocity structure, as well as improving our physical understanding of the geologic basis for the lateral variability observed in these tomography studies. These comparisons as well as mechanisms for wider dissemination of these geophysical databases should be discussed in the tomography workshop.

3. The Bayesian kriging methodology developed by LLNL and SNL may hold promise for representing surface wave travel-time and amplitude correction surfaces for CTBT stations similar to the better established body wave correction surfaces. Super-resolution experiments in which kriged surface wave maps built on tomographic base maps should be performed and the resulting maps should be evaluated using the same criteria as applied to the base maps.

\section{Knowledge Transfer:}

Although workshops such as this one appear to be an effective way to exchange information and define future goals, they are somewhat too large and too broadly defined to allow consensus to be reached on substantive technical issues. Smaller working groups are better suited to deal with specific technical goals and adjudicate between competing methodologies, maps, models, etc. We recommend that this workshop be followed-up by at least three future working group meeting on the U.S. use of surface waves for CTBT monitoring. For operational utility, the schedule of these meetings should be tied to both the DOE Knowledge Base and the NDC testing and evaluation schedules. The goal of the meetings will be to get significant results into the Knowledge Base by the year 2000. The National Laboratories (LLNL, LANL, and SNL), in conjunction with AFTAC, will define the formats for data transmission and metadata. The principal result will be group velocity maps, but could also include group velocity curves, station correction surfaces and, kriged super-resolution correction surfaces.

Membership of the working group should include representatives from CTBT research groups funded to produce or evaluate group velocity maps at and below $20 \mathrm{~s}$ period and should be kept small in number $(<\sim 10)$.

A proposed schedule for the first three working group meetings is as follows.

1) September 1998, Santa Fe (in conjunction with the 20th Annual CTBT Seismic Symposium)

Topics: Status report of efforts by various groups. Data exchange issues and solutions, data averaging and clustering. Systematic bias and error analysis.

Discussion of future research and working group plans.

2) April 1999, LLNL, CA

Topics: Tomographic map comparison. Establishment of performance criteria. Identification of problem regions.

3) September 1999, Sandia NL (sometime after the 21st Annual CTBT Seismic Symposium) 
Topics: Production of surface wave maps for CTBT use that embody the state of the art and are in a suitable form for an operational system. Proposal for incorporation into DOE knowledge base along with metadata. Development of a. proposed plan for updates as maps are improved.

The Knowledge Base is expected to undergo revisions as a result of testing and evaluation by DOE and the U.S. NDC and as new data and research results become available. We would expect that as this occurs there may be future workshops to incorporate these updates.

Acknowledgments: The success of this workshop is primarily due to the enthusiastic and unselfish cooperation the participants. We especially would like to thank the groups that participated in the dispersion measurement tests and the dispersion tomography comparisons. Comments by Keith Mclaughlin, Jeff Stevens and Howard Patton improved this summary repont. Special thanks to Artie Rodgers for making Figures 1 and 3. W. R. Walter's work was performed under the auspices of the U.S. Department of Energy by Lawrence Livermore National Laboratory under contract W-7405-ENG-48. 


\section{Appendix}

\section{Agenda of Workshop on the U.S. Use of Surface Waves in Monitoring a CTBT}

THURSDAY MARCH 19, 1998

Morning Session:

Chair: Mike Ritzwoller

8:00 M. Ritzwoller, B. Walter: Welcome, Introduction, Logistics, and Overview of Workshop

Session 1: Regional Dispersion Measurement Comparison

(data selection, measument methodology, error assessment)

(each speaker plans to talk for only 10 minutes (max 8 viewgraphs) to allow for: questions and answers from floor)

8:20 A. Levshin Measurement at CU and general comments

8:35 D. McNamara Measurement at LLNL

8:50 L. Jones Measurement at LANL

9:05 R. Hermann Measurement at SLU

9:20 J. Stevens Global and IDC Measurements

9:35 B. Walter Comparison of dispersion curves supplied by

9:50 Discussion

researchers for 10 presclected event-station pairs*

10:15-10:30 Break

Session 2: Regional Dispersion Map Comparison

(data coverage, inversion technique, resolution estimation)

(each speaker plans to talk for only 10 minutes (max 8 viewgraphs) to allow for questions and answers from floor)

10:30 D. McNamara North Africa and the Middle Fast

10:45 R. Herrmann Saudi Arabia and Surroundings

11:00 M. Ritzwoller Eurasia

11:15 J. Stevens World

11:30 A. Rodgcrs Comparison of 20 s Rayleigh wave group velocity maps

11:45 Discussion supplied by researchers for $10-50 \mathrm{~N}, 25-100 \mathrm{E}$ region*

12:15-1:30 Lunch 
Afternoon Session:

Chair: Bill Walter

Session 3: Customers for surface wave research

1:30 D. Russell AFTAC's interest in surface waves

2:30 R. Keyser DOE Knowledge Base and surface wave information

3:00 C. Meade NPTO's interest in surface waves

3:15 Discussion

$3: 30-3: 45 \quad$ Break

Session 4: Use and Evaluation of group velocity maps

3:45 J. Stevens Using group velocity maps and the M.s $\mathrm{mb}$ discriminant

4:15 D. Harkrider Evaluation of group velocity maps

Session 5: Dispersion Complications, Problems and Future Directions

4:30 A. Levshin Group source time shifts

4:45 E. Larson Anisotropy and global phase velocity estimation

5:00 M. Ritzwoller Mislocation, anisotropy, off-great-circle

5:15 Discussion

propagation and regional tomography

5:30 Adjourn for the day.

FRIDAY MARCH 20, 1998

Morning Session:

Chair: Bill Walter

8:00 M. Ritzwoller, B. Walter Opening Remarks

Session 6: Source Characterization

8:15 H. Patton LANL source characterization

8:30 A. Levshin Moment tensor, depth estimation for Lop Nor

8:45 Discussion

Session 7: Velocity models and surface wave dispersion maps

9:00 D. McNamara Pn tomography results for Eurasia

9:15 B. Walter Evaluation of MidEast/North Africa velocity models

9:30 M. Ritzwoller Eurasian crustal/lithospheric model, potential use for CTBT

9:45 R. Engdahl Eurasian upper mantle

10:00 Discussion 


\section{0:15-10:30 Break}

Session 8: Closing Discussion, Preparation of Comments for Workshop Report

10:30 - noon Discussion

noon Adjourn meeting

* The idea for these two sessions is to ask participants to supply dispersion curves for 10 pre-selected event station pairs in the 10-50 N latitude, 25-100 E longitude region as well as their 20 s Rayleigh wave tomographic maps in this region and then compare them.

The purpose is not to choose which institution's maps or measures are more correct, but to understand the causes of any differences the comparisons reveal and provide a common basis for discussion. 


\section{Events, Stations and Paths for Comparison}

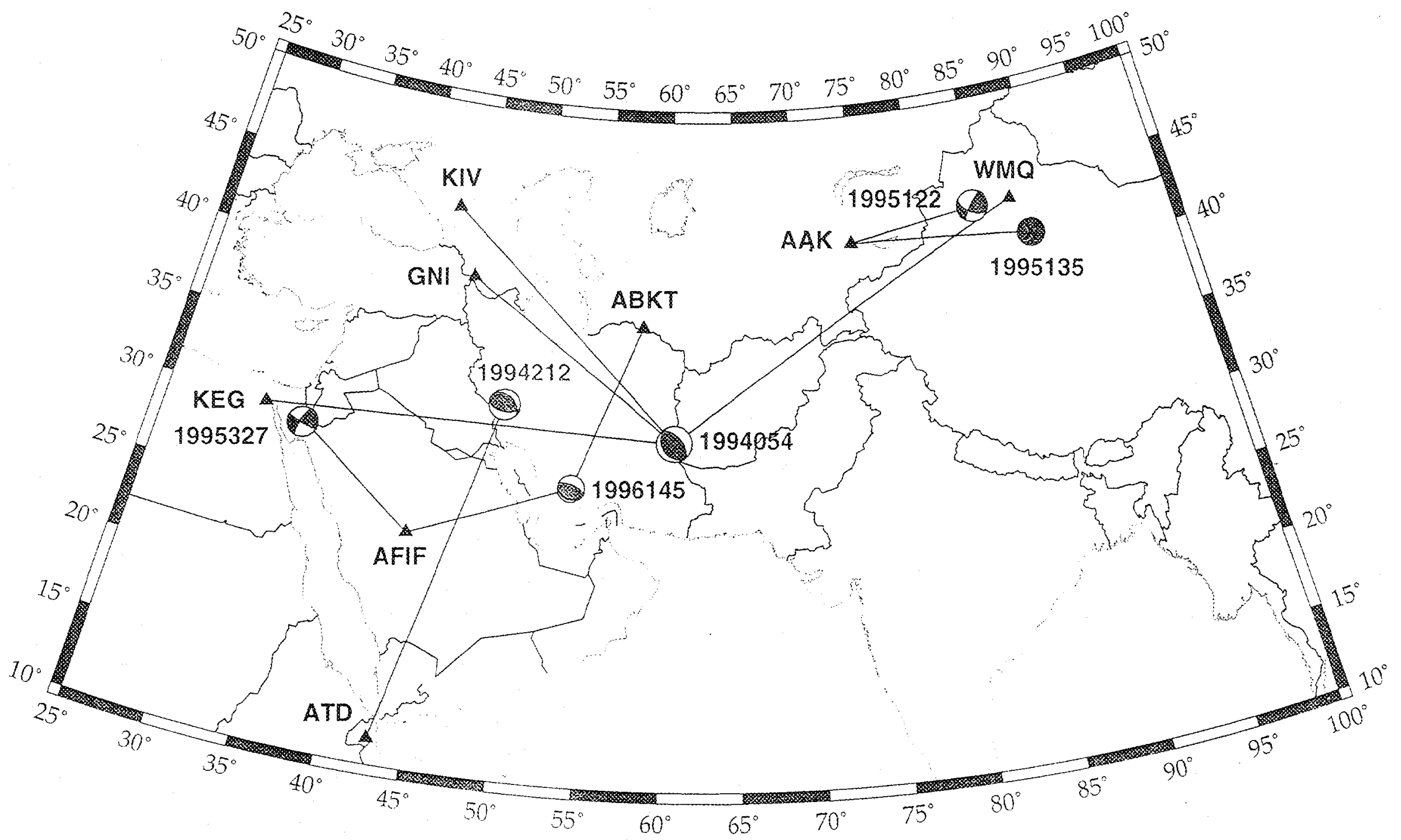

Fig. 1. Map showing focal mechanisms. paths and recording stations for the 10 event-station pairs measured and compared at the workshop. These 10 paths were chosen under the following considerations:

1. Fit within box shown, with emphasis on regional and near teleseismic distance $(800-2800 \mathrm{~km})$ shorter period measures in a complex region

2. Have a range of Harvard CMT mechanisms, and cover a range of magnitudes $m b=4.9$ to 6.1

3. Some paths likcly to already be part of each Institutions measures

4. Unintentional test - GNI and ATD waveforms have 40 to 50 second timing exrors 

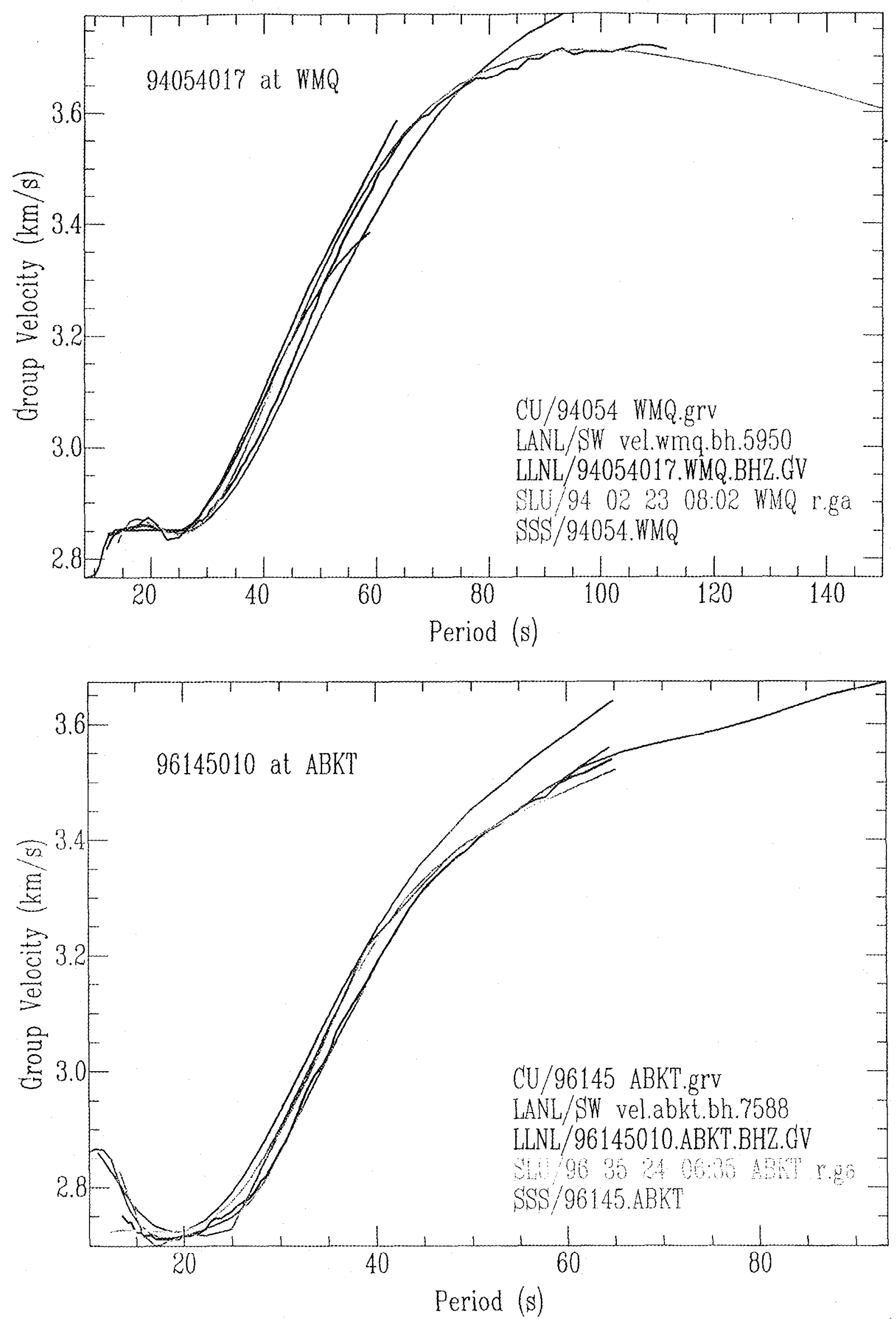

Fig. 2. Examples of group velocity curve measurements by 5 different institutional groups.

Differences occur at choices of short and long period cuts, and where velocity changes rapidly. 


\section{Measures of Differences between Group Velocity Curves}


Fig. 3. Statistical measures of the differences observed between the 5 institutions measurements. Top shows standard deviation from the mean for all paths by period. Different cuts result in different numbers of residuals. Bottom shows the maximum difference for each specific group velocity curve. 